\title{
Polar magnetic fields and coronal holes during the recent solar minima
}

\author{
Giuliana de Toma \\ High Altitude Observatory \\ National Center for Atmospheric Research \\ P.O. Box 3000, Boulder, CO 80307-3000 \\ email: detoma@ucar.edu
}

\begin{abstract}
The slow decline of solar Cycle 23 combined with the slow rise of Cycle 24 resulted in a very long period of low magnetic activity during the years 2007-2009 with sunspot number reaching the lowest level since 1913. This long solar minimum was characterized by weak polar magnetic fields, smaller polar coronal holes, and a relatively complex coronal morphology with multiple streamers extending to mid latitudes. At the same time, low latitude coronal holes remained present on the Sun until the end of 2008 modulating the solar wind at the Earth in corotating, fast solar wind streams. This magnetic configuration was remarkably different from the one observed during the previous two solar minima when coronal streamers were confined near the equator and the fast solar wind was mainly originating from the large coronal holes around the Sun's poles. This paper presents the evolution of the polar magnetic fields and coronal holes during the past minimum, compare it with the previous minima, and discuss the implications for the solar wind near the Earth. It also considers the minimum of Cycle 23 in an historical perspective and, in particular, compares it to the long minima at the turn of the 19th century.
\end{abstract}

Keywords. Sun: sunspots, corona, solar wind

\section{Introduction}

The solar minimum between Cycles 23 and 24 was the longest and deepest minimum in about 100 years. The northern hemisphere reached solar minimum conditions already in 2006. In 2007-2008, sunspots continued to emerge, albeit at a very low level, preferentially in the southern hemisphere. In 2009 magnetic activity remained very low, with most of the active regions emerging on the Sun belonging to the new Cycle 24. The years 2008 and 2009 were extremely quiet years, with spots present on the Sun less than $30 \%$ of the time (Figure 1). Because magnetic flux emergence was so low for an extended period of time, it is not easy to identify a single point in time as the minimum between Cycle 23 and 24 but the periods in August 2008 and August 2009 stand out as particularly quiet times when spots were absent from the Sun for over 30 consecutive days.

This extremely quiet minimum differed in many ways from other recent minima for which we have a good observational record and changed our idea about solar minima. Not only were sunspot activity, CMEs, and flares extremely low during the past minimum, the solar wind had anomalously low densities and magnetic fields, both at the poles and in the ecliptic (e.g., McComas et al. 2008, Issautier et al. 2008, Smith \& Balogh 2008, Jian et al. 2010). The interplanetary magnetic field measured at 1 AU continued to decline in 2006-2009 and by 2009 had decreased by 30\% compared to 1996, reaching its lowest value ever recorded in 50 years of observations (e.g., Wang et al. 2009, Tsurutani et al. 2011). However, in spite of the low magnetic activity, the corona and heliosphere remained relatively complex throughout most of the minimum phase. 

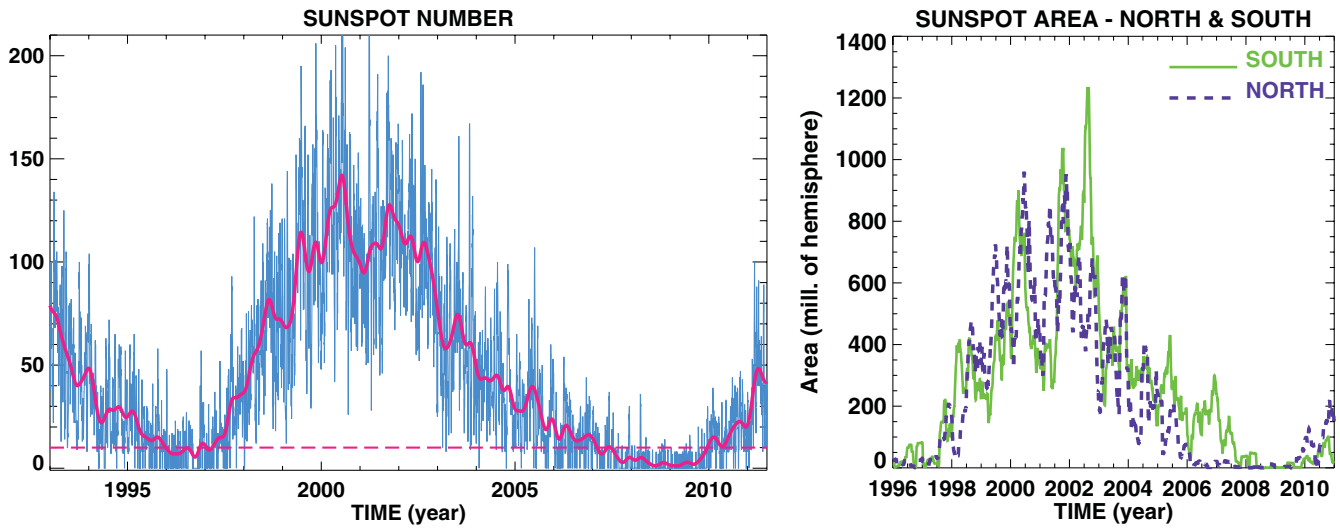

Figure 1. (Left) Daily sunspot number and 3-rotation averages. The minimum between Cycles 23 and 24 corresponds to a long period of extremely low magnetic flux emergence with sunspot activity lower than in 1996. (Right) Sunspot area averaged over 3 rotations for the northern and southern hemisphere. The north reached solar minimum conditions before the south, and Cycle 24 activity started first in the north and later in the south.
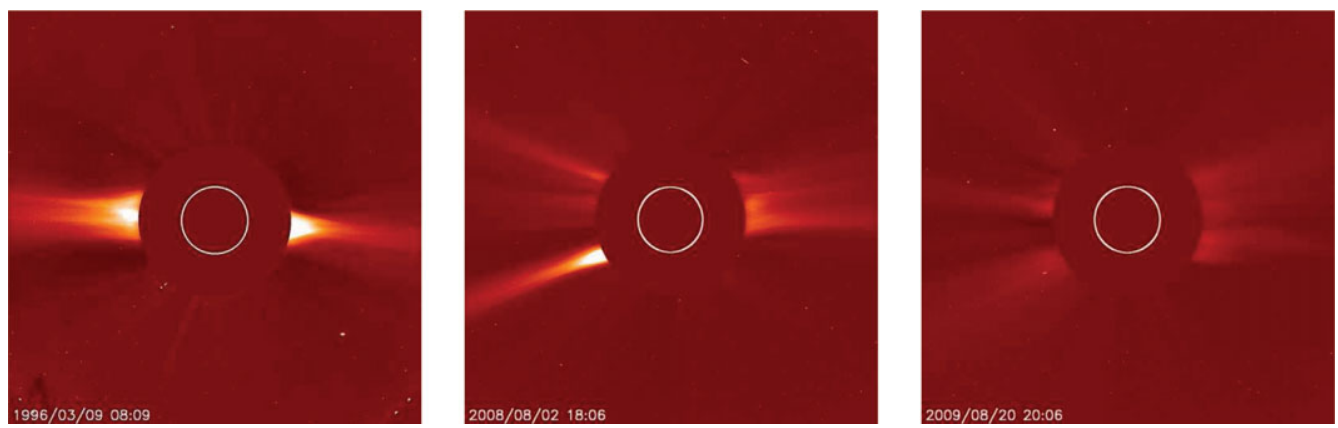

Figure 2. LASCO images showing the solar corona in visible light in March 1996 (left) August 2008 (center) and August 2009 (right). Even during the extremely quiet periods in August 2008 and August 2009 the shape of the solar corona was more complex than in 1996.

Sections 2 and 3 discuss the properties and evolution of the solar corona and solar wind during the minimum of Cycle 23 and the role of weak polar magnetic fields at the photosphere in determining the complexity of the corona and heliosphere during this very quiet time. Section 4 examines other low solar minima in the historical sunspot record, while conclusions are given in Section 5 .

\section{The Solar Corona during the Minimum of Cycle 23}

The shape and structure of the solar corona and heliosphere were noticeably different during the minimum of Cycle 23 compared to other recent minima, and, interestingly, were not as simple as in 1996 or 1986 (e.g., Gibson et al. 2009, Luhmann et al. 2009, de Toma et al. 2010, Petrie et al. 2010). The solar corona was still quite complex in 2007 and 2008, even if sunspot activity was below the levels seen in 1986 and 1996, and slowly evolved toward a simpler structure in 2009, but it never reached a "dipolar" configuration with coronal streamers confined to a narrow band around the solar equator. Multiple streamers and pseudo-streamers (Wang et al. 2009, Riley et al. 2010, Gibson et al. 2011) remained present on the Sun even during times of extremely low magnetic activity (Figure 2). The heliosphere was also more complex for a significant fraction 
of the Cycle 23 minimum (e.g., Tokumaru et al. 2009, 2010) and only in 2009 did the heliospheric current sheet assume a relatively "flat" configuration, more typical of solar minimum (de Toma et al. 2010b).

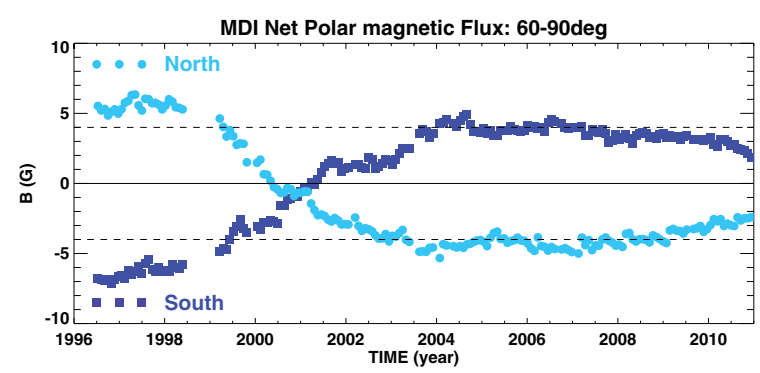

Figure 3. (Top) Polar magnetic flux as measured by SOHO/MDI. The strong decrease in the net polar magnetic flux during the past minimum was also observed at ground-based solar observatories, such as at Kitt Peak and Wilcox Solar Observatory. The polar magnetic fields remained almost constant between 2004 and 2008, and in mid 2009, with the increase in Cycle 24 activity, started to slowly decline. (Bottom) Interplanetary magnetic field (IMF) averaged over 27 days. In contrast to the polar fields, the IMF continued to slowly decline in 2006-2009.

The more complex morphology of the solar corona during the past minimum was largely because of the weak polar magnetic fields at the photosphere (e.g., Sheeley 2008). One of the striking observational features of the late declining phase and extended minimum of Cycle 23 was the low value of the polar magnetic flux. In 2004-2009, the net polar magnetic flux above $60^{\circ}$ latitude was remarkably stable and about $40 \%$ lower than observed during the previous two minima as illustrated in Figure 3 (see also Svalgaard \& Cliver 2007, Sheeley 2008, Wang et al. 2009). Thus, the continuous decline observed in the interplanetary magnetic field was not related to changes in the polar regions, but rather was caused by changes in the "open" magnetic flux from lower latitude sources, as we will discuss below.

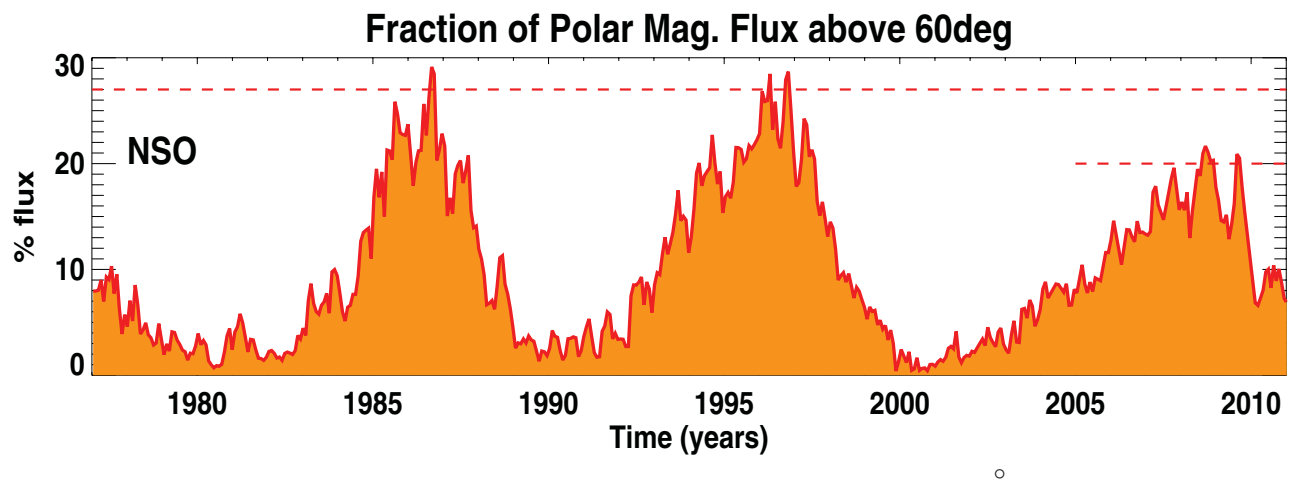

Figure 4. Fraction of the total magnetic flux in the polar region above $60^{\circ}$ latitude as measured at NSO/Kitt Peak using KPVT and SOLIS magnetograms. The ratio of the net polar magnetic flux to the total magnetic flux during the minimum of Cycle 23 was significantly lower than during the previous two minima.

The lower value of the polar magnetic field resulted in global coronal field with a weaker polar dipole moment $(l=1, m=0)$ while higher order moments did not change significantly. Figure 4 gives the ratio of the net polar magnetic flux -which is directly related to the strength of the polar dipole component- to the total unsigned magnetic flux at the 
photosphere, as measured at NSO/Kitt Peak. This ratio always reaches its maximum value near solar minimum, when polar magnetic fields are strongest and activity at mid and low latitudes is low. During the minimum of Cycle 23 the ratio of the polar to the total magnetic flux decreased significantly compared to the previous two minima. As a result, even if the polar dipole remained the dominant coronal field component (Petrie et al. 2010), the ratio of the polar dipole to the equatorial dipole and to the quadrupole moment decreased during the minimum in 2008-2009 relative to 1996 and 1986 minima, when the polar dipole was larger (e.g., Wang et al. 2009, Petrie et al. 2010, DeRosa et al. 2011). This, in turn, resulted into a more complex coronal configuration and in a tilted and warped heliospheric current sheet and allowed mid- to low-latitude streamers and pseudo-streamers to remain present even during times of extremely low activity (Gibson et al. 2011 and references therein).

\subsection{Coronal Holes}

The special solar minimum configuration at the end of Cycle 23 with very weak polar fields, and a weak polar dipole, had significant implications for the organization and evolution of coronal holes on the Sun and, consequently, for the solar wind. Figure 5 shows EUV synoptic maps of the Sun in the FeXII $195 \AA$ lines where coronal holes appear as dark regions in the corona. The top two panels show the EUV corona in 1996 during the minimum of Cycle 22 when polar fields were strong. The bottom two panels show the corresponding coronal hole maps derived using a combination of magnetograms and four different EUV wavelengths (de Toma \& Arge 2005, de Toma 2010). In 1996, coronal holes were organized in two large polar coronal holes that extended to or below $50^{\circ}$ latitude. The area of each polar hole was about $7-8 \%$ of the Sun's surface while low-latitude coronal holes were small or absent (Harvey \& Recely 2002, de Toma 2010).
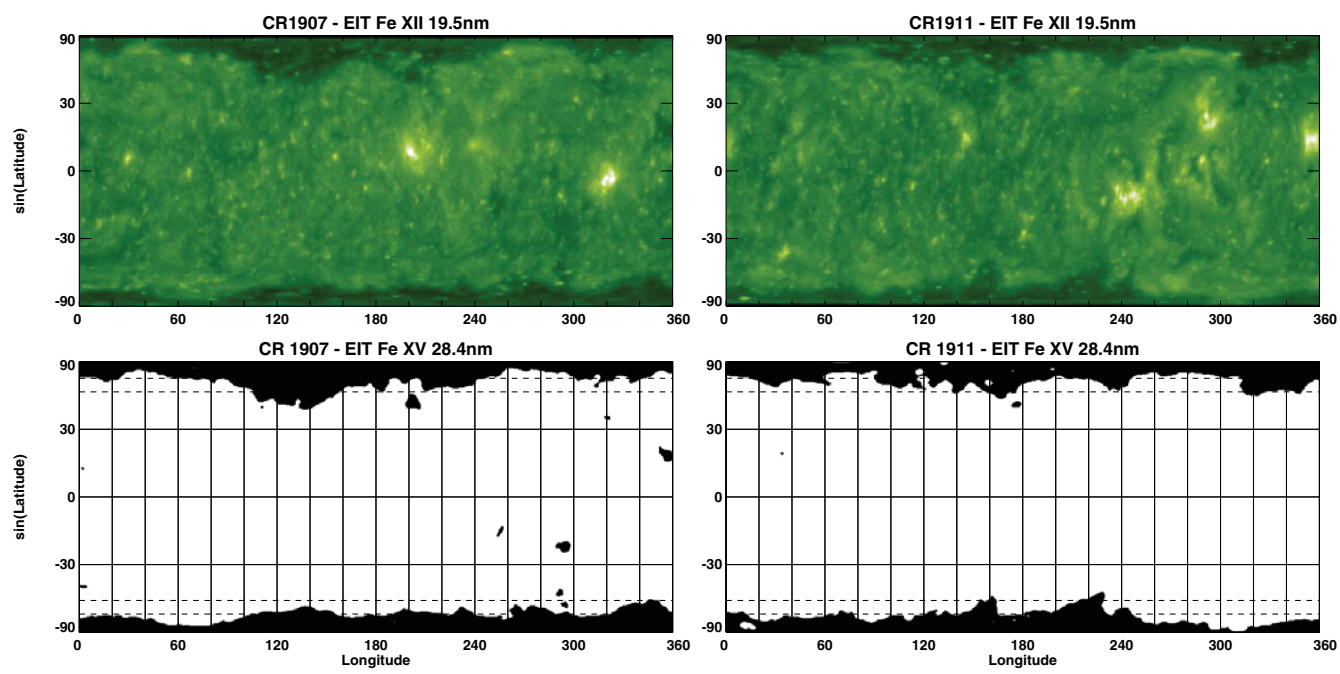

Figure 5. (Top) EUV synoptic maps of the Sun constructed from EIT Fe XII images where coronal holes appear as dark regions in the corona. The maps are in sin(latitude) and thus have equal area pixels. The two Carrington Rotations shown are CR 1907 in March 1996 and CR 1911 in July 1996 during the minimum of Cycle 22. (Bottom) Corresponding coronal hole maps. The dashed lines mark $50^{\circ}$ and $60^{\circ}$ latitude.

Figure 6 shows EUV synoptic maps of the Sun and the corresponding coronal hole maps for the minimum of Cycle 23. Polar coronal holes were significantly smaller, by 20-40\%, than observed during the previous minimum (de Toma 2010) and mostly confined above 

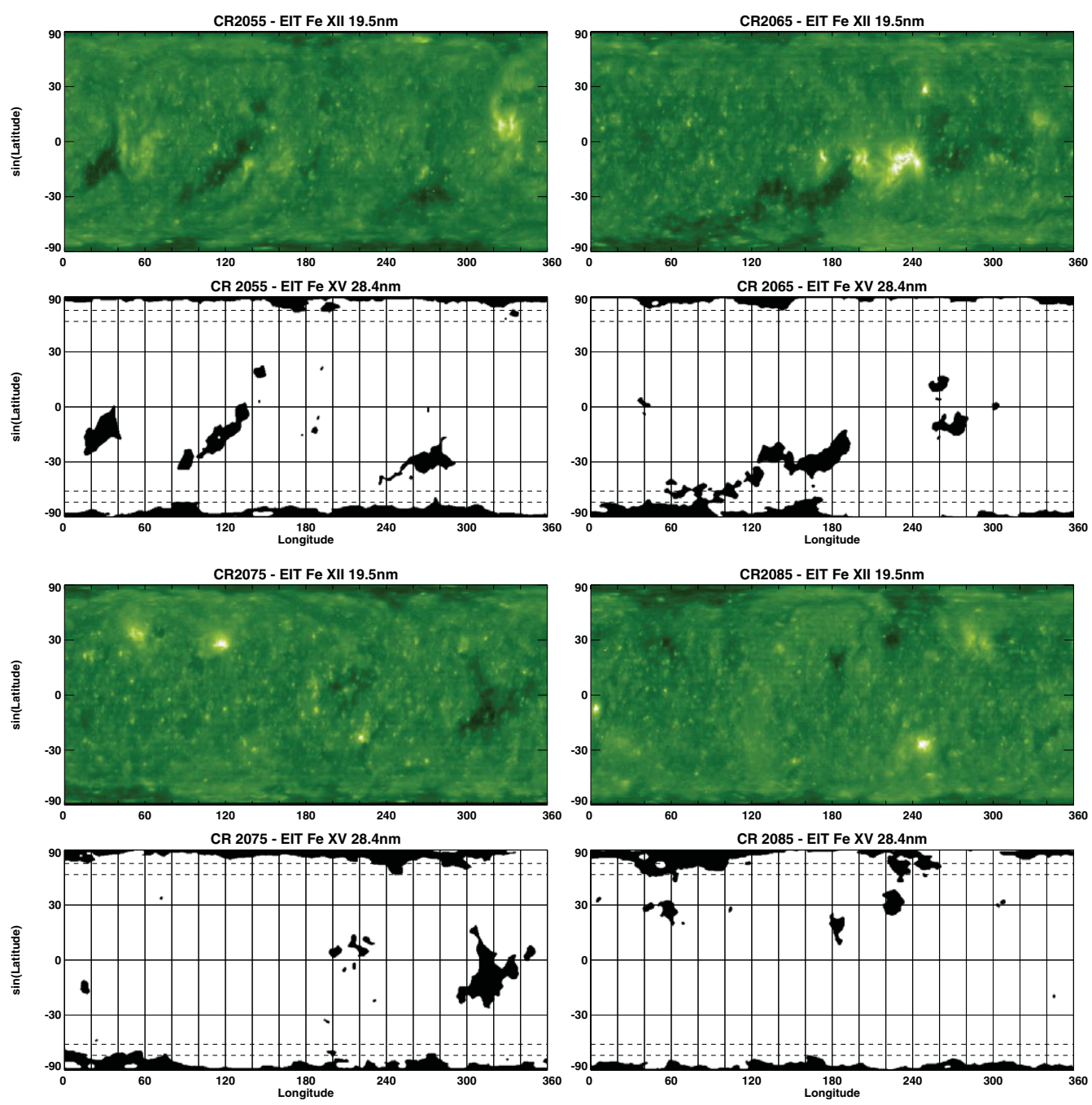

Figure 6. EUV synoptic maps of the Sun and corresponding coronal hole maps as in Figure 5 but for CR 2055 in April 2007, CR 2065 in January 2008, CR 2075 in October 2008, and CR 2085 in July 2009 during the extended minimum at the end of Cycle 23.

$60^{\circ}$ latitude. At the same time, isolated coronal holes of significant size were present on the Sun between $\pm 30^{\circ}$ latitude. These low-latitude coronal holes, commonly seen during the declining phase of the solar cycle, persisted into the minimum phase of Cycle 23 and continued to be important sources of fast solar wind at the Earth in 2006-2008 (Gibson et al. 2009, Luhmann et al. 2009, Lee et al. 2009, Abramenko et al. 2010, de Toma 2010). They were long-lived and produced weak to moderate recurrent geomagnetic storms (Tsurutani et al. 2011). As a result, the solar wind speed maintained some of the 9-, 13.5-, and 27-day periodicities typically observed during the declining phase (Emery et al. 2009, 2010). The same periodicities were also seen in other observations, including auroral and geomagnetic indices, radiation belt electron flux (Gibson et al. 2009), and even propagated down into the upper atmosphere (Thayer et al. 2008, Lei et al. 2010). At the end of 2008, these large, low-latitude coronal holes started to close down and finally disappeared in early 2009 (de Toma 2010). In 2009, small and transient, mid-latitude coronal holes formed in the remnants of the first Cycle 24 active regions. These small 

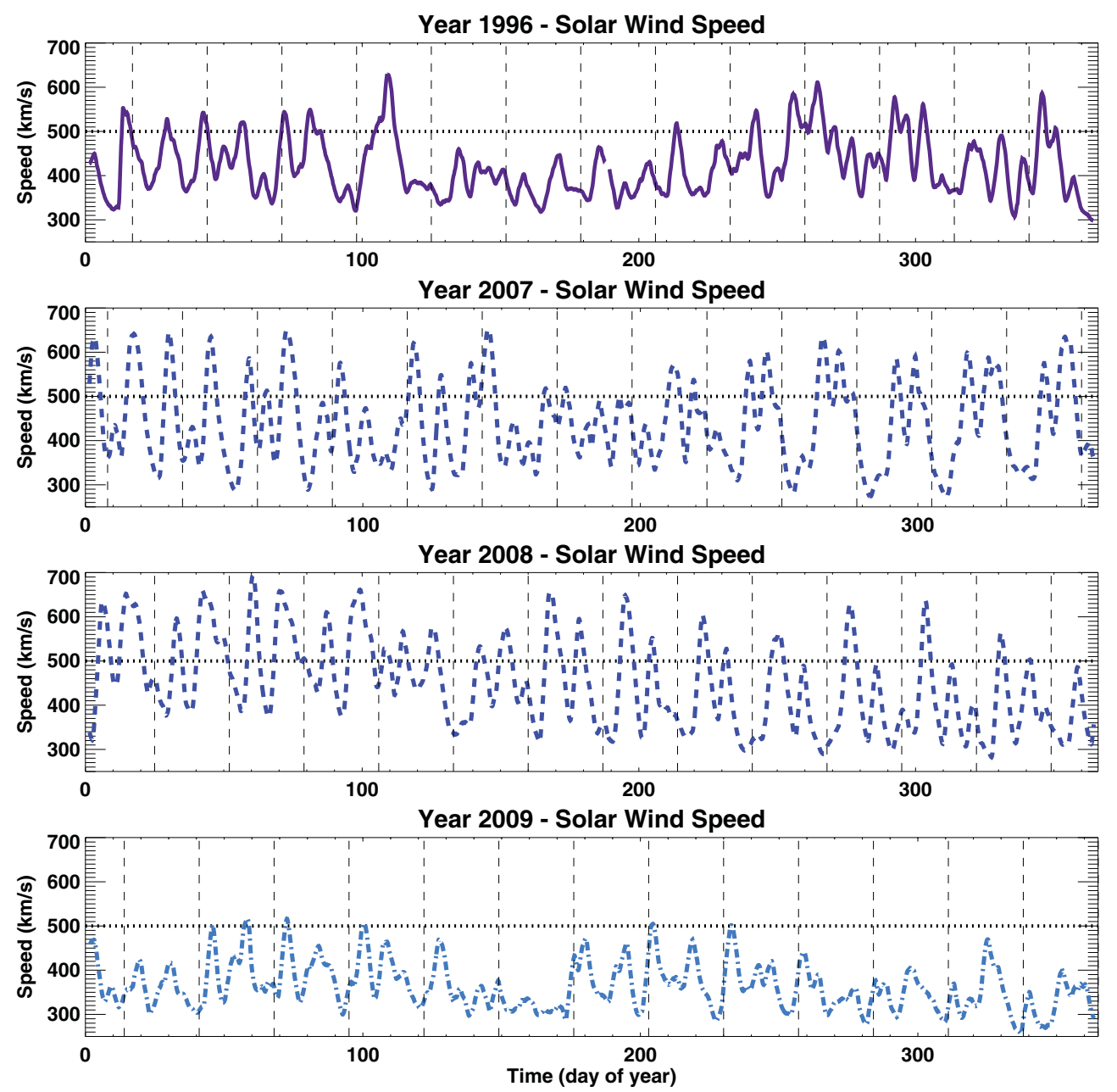

Figure 7. Solar wind velocity time series for the years 1996, 2007, 2008, and 2009. Hourly solar wind speeds have been averaged over 3 days.

and short-lived coronal holes were not important sources of the solar wind at the Earth, which originated mostly from the edges of the polar coronal holes during this time.

The persistence of large low-latitude coronal holes in 2007 and 2008 was possible because of the particular magnetic flux distribution at the Sun. Not only polar fields were weaker than in 1996 but the predominantly unipolar regions around the poles were confined to a smaller area at high latitudes. This gave rise to smaller polar coronal holes and made it easier for quasi-unipolar magnetic regions at low-latitude to "open" into the heliosphere. Numerical experiments using potential field source surface extrapolations where the photospheric polar magnetic fields are artificially modified (e.g., Wang et al. 2009, Luhmann et al. 2009, de Toma \& Arge 2010) show that increasing the flux density near the poles in 2008 results in smaller low-latitude coronal holes, while decreasing it in 1996, makes it possible to form low-latitude coronal holes. de Toma \& Arge (in preparation) find that not only the strength of the polar fields is important, but that the magnetic flux distribution near the poles and the latitudinal extent of the quasi-unipolar polar regions also play an important role in determining the distribution and size of coronal holes on the Sun at both high and low latitudes. 


\section{Solar Wind during the Minimum of Cycle 23}

Figure 7 shows the time series of solar wind speed for the years 1996, 2007, 2008, and 2009. In 2007 and 2008, the yearly mean solar wind speed was $440 \mathrm{~km} / \mathrm{s}$ and $448 \mathrm{~km} / \mathrm{s}$, respectively, similar to the $423 \mathrm{~km} / \mathrm{s}$ observed in 1996. Nonetheless, in 2007 and 2008 the solar wind was more structured than in 1996 with regular high-speed streams, and fast solar wind was more commonly seen at the Earth. Speeds above $500 \mathrm{~km} / \mathrm{s}$ were observed $29 \%$ and $33 \%$ of the time in 2007 and 2008 in hourly averaged solar wind data compared to $17 \%$ of the time in 1996 . This fast wind originated from the large and long-lived, isolated coronal holes discussed above and gave origin to the prominent high-speed tail in the velocity distribution indicated by the arrow in Figure 8 (de Toma 2010). In 2009, with the disappearance of the low-latitude coronal holes, there was an abrupt change in the solar wind speed at the Earth. The mean solar wind velocity dropped to an yearly value of $365 \mathrm{~km} / \mathrm{s}$, a $20 \%$ decrease from 2008 . Solar wind speeds above $500 \mathrm{~km} / \mathrm{s}$ were seen at Earth only $4 \%$ of the time and the high-speed velocity tail in the solar wind distribution disappeared. At the same time, the interplanetary magnetic field continued to slowly decline, as these low-latitude source regions of fast wind closed down.

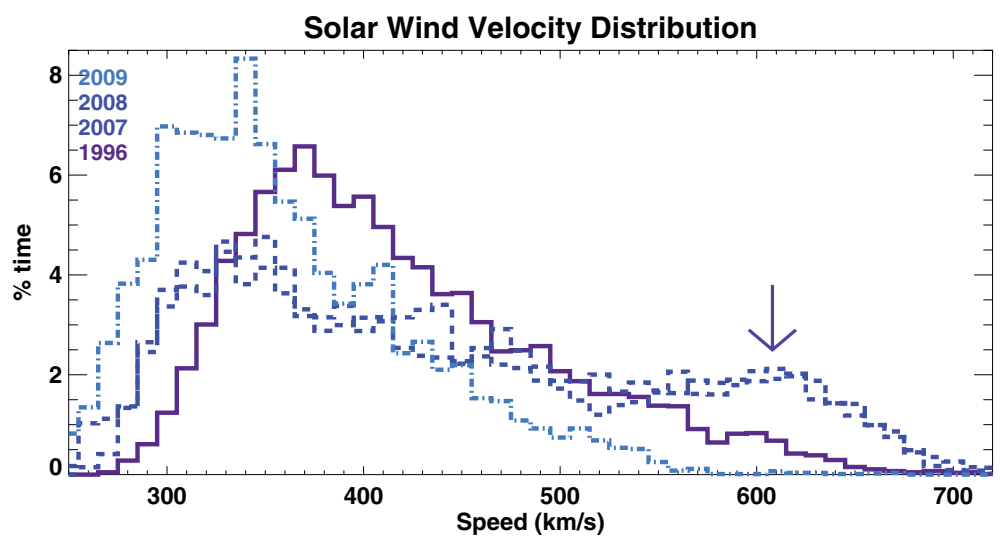

Figure 8. Solar wind velocity distributions for hourly solar wind speeds for the year 1996, 2007, 2008, and 2009. In 2007 and 2008, the velocity distribution is almost bimodal with a secondary peak at about $600 \mathrm{~km} / \mathrm{s}$. This high-velocity tail disappears completely in 2009 and the peak of the distribution shifts towards lower speeds.

The changes in the solar wind speed during the minimum of Cycle 23 had a very important effect on the Earth's radiation belts which remained elevated in 2007 and 2008, when solar wind speeds above $500 \mathrm{~km} / \mathrm{s}$ were relatively common (Gibson et al. 2009), and almost completely vanished in 2009, when velocities dropped below $500 \mathrm{~km} / \mathrm{s}$ (D. Baker, private communication). Geomagnetic activity also reached its lowest value in 2009 (e.g., Emery et al. 2010, Tsurutani et al. 2011) when the solar wind magnetic field and speed reached their minimum level.

\section{Solar Minima in the Historical Record}

While this minimum clearly differed from the other solar minima during the Space Age, the historical sunspot record indicates that this was not an unusual minimum and that very long and deep minima have occurred several times in the past. In particular, the minima at the end of the 19th century and beginning of the 20th century were as long or longer than the quiet period in 2007-2009. Unfortunately, we do not have direct 
solar wind observations for that period and only very limited solar observations besides sunspots.
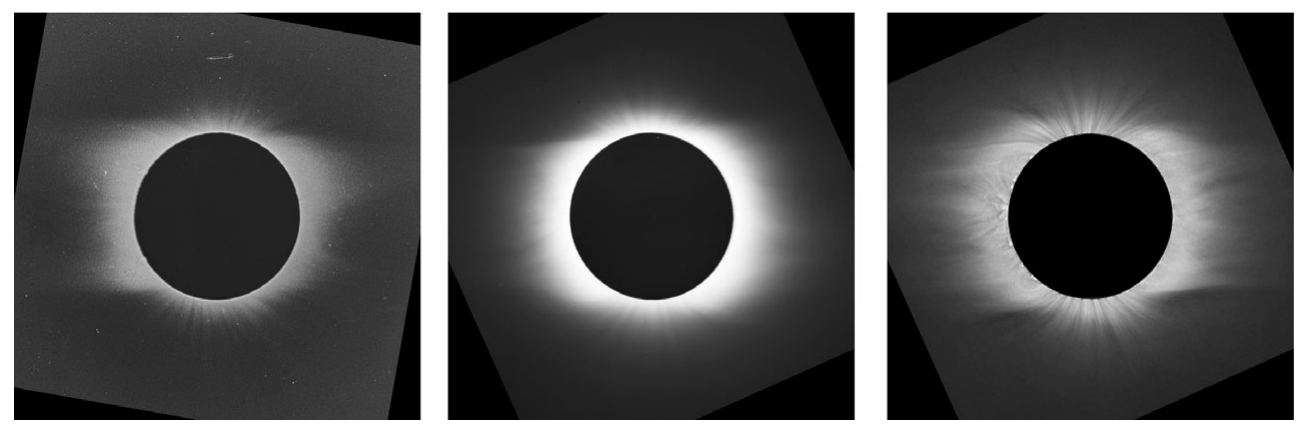

Figure 9. Photographs of the total solar eclipses in December 1889 in French Guiana (left) and in June 1901 in Sumatra (center). The right panel shows a composite image for the 1901 eclipse obtained by combining multiple frames with different exposures (courtesy of M. Druckmüller).

Routine monitoring of the solar corona in broad-band visible light started in the '60s at Mauna Loa Solar Observatory (Fisher et al. 1981) while coronal observations in the FeXIV emission line go back to the '40s (Billings 1966). Before this time, we only have solar eclipses available to infer the morphology of the corona (e.g., Judge et al. 2010). However, total eclipses are rare and short in duration and give us only a snapshot of the solar corona. They occur approximately once in 18 months, and not always at easy-to-reach locations, therefore, they cannot give us information about coronal evolution on time scales of less than a year, yet, they are useful to gain insight to the coronal morphology at different phases of the solar cycle. At the High Altitude Observatory in Boulder, Colorado, we have a collection of old eclipse photographs, http: //mlso.hao.ucar.edu/mlso_eclipses.html, that goes back to 1869. Figure 9 shows two of such photographs for the years 1889 and 1901, during the solar minima at the end of Cycle 12 and 13. These minima with 212 and 287 days without spots had similar sunspot activity to the years 2008 and 2009. It is, thus, interesting to examine the coronal shape at the times of these old eclipses. Multiple photographs were taken with different exposure times which can be combined to obtain composite images of the solar corona to better display the streamer structure (see the right panel of Figure 9 and Judge et al., 2010). The eclipse images in 1889 and 1901 in Figure 9 reveal a solar corona far from a simple dipolar structure and show that multiple coronal streamers existed for at least part of these long solar minima, suggesting weak polar magnetic fields. Interestingly, the $a a$ index for these minima indicates a low level of geomagnetic activity, and a Fourier analysis of the index shows periodicities at 7-, 9-, and 13.5-days, which might indicate the presence of recurrent, low-latitude coronal holes. Thus, these minima at the turn of the 19 th century may have been similar to the recent solar minimum.

\section{Conclusions}

The long and deep minimum at the end of Cycle 23 was the lowest and longest minimum in about a century. It differed in many ways from the other solar minima observed during the Space Age for which we have a good observational coverage both at the Sun and in the heliosphere. This gave us an opportunity to advance our understanding of solar minima and, in particular, of deep minima. It also changed our ideas about the corona and heliosphere at solar minimum. 
In 2007-2009, the regions around the solar poles dominated by one magnetic polarity were covering a smaller area than in 1996, had generally weaker magnetic flux density, and were less unipolar (de Toma \& Arge, in preparation). All these factors contributed to give weaker polar fields and, in turn, a weaker polar dipole moment. This special magnetic configuration during the Cycle 23 minimum led to a coronal morphology far from the typical dipolar structure observed during the minima in 1986 and 1996. Polar coronal holes were smaller and quasi-unipolar magnetic regions at lower latitudes were able to open to the heliosphere and survive longer on the Sun, forming large and persistent, low-latitude coronal holes during the years 2007-2008. At this time, the ratio of the polar dipole to higher order moments was significantly lower than during the previous minimum in 1996. As low activity continued, little new magnetic flux emerged on the Sun while existing low-latitude magnetic fields continued to disperse and decay under the effect of differential rotation, meridional circulation, and random convective motions. This resulted in a decrease of the higher order components of the Sun's global magnetic field. In 2009, low-latitude coronal holes finally closed down and the heliosphere relaxed to a simpler two-sector structure, even if not as simple as during the preceding minima, when polar fields were stronger (Gibson et al. 2011). This had important implications for the solar wind impinging on the Earth. The low-latitude coronal holes present on the Sun in 2007 and 2008 were sources of recurrent, high-speed streams that triggered moderate to low geomagnetic activity. In 2009, these low-latitude sources of fast solar wind went away and the solar wind at the Earth was coming mostly from the edges of the relatively small polar coronal holes. This is when the magnetic field carried by the solar wind, the solar wind speed, and geomagnetic activity all reached their lowest level, showing a time delay between the minimum observed at the photosphere and in the heliosphere (Tsurutani et al. 2011).

The differences between Cycle 23 minimum and other recent minima discussed above show that solar minima are not all the same and that the Sun can have different magnetic configurations even during very quiet times, with significant consequences for the heliosphere. What ultimately determines the structure of the corona and heliosphere, even at solar minimum, is the magnetic flux distribution at the Sun. Therefore, sunspot number, while a very good indicator of the global activity level, cannot describe the 3D corona and heliosphere.

While Cycle 23 minimum was markedly different from previous minima observed during the Space Age, the historical records of sunspots, solar eclipses, and geomagnetic activity indicate that the recent minimum was not peculiar and similar long and deep minima have occurred in the past. In particular, the low minima at the turn of the 19th century appear to resemble the recent minimum.

Finally, the extremely low magnetic activity reached during the end of 2008 and 2009 gave us some insight on what physical conditions could have existed during grand minima (Schrijver et al. 2011). The length of Cycle 23 minimum, combined with the very low magnetic flux emergence, allowed magnetic fields present on the Sun the time to diffuse and decay to smaller and smaller scales in a manner that we had not observed before with modern instrumentation (McIntosh et al. 2011).

\section{Acknowledgements}

I deeply thank my collaborators C.N. Arge, S. Gibson, B. Emery, J. Kozyra, P. Judge, and J. Burkepile for the long and useful discussions on the prolonged solar minimum at the end of Cycle 23. I also thank the IAU organizing committee for partially supporting my trip to the IAU Symposium 286 in Mendoza. 
The National Center for Atmospheric Research is sponsored by the National Science Foundation. This work was partially supported by the LWS NASA Grant \# NNH05AA49.

\section{References}

Abramenko, V., Yurchyshyn, V., Linker, J., Mikić, Z., Luhmann, J. G., \& Lee, C. O., 2010, ApJ, 712,813

Billings D. E., 1966, A Guide to the Solar Corona, New York, Academic Press

DeRosa, M. L., Brun, A. S., \& Hoeksema, J. T., 2011, Astrophysical Dynamics: From Stars to Galaxies, Proceedings of the International Astronomical Union, IAU Symposium 271, 94

de Toma, G. \& Arge, C. N., 2005, ASP Proceedings of the NSO Workshop 22: Large-Scale Structures and Their Role in Solar Activity, 251

de Toma, G. \& Arge, C. N., 2010, Twelfth International Solar Wind Conference, AIP Conference Proceedings vol. 1216, 679

de Toma, G., Gibson, S. E., Emery, B. A., \& Kozyra, J. U., 2010, Twelfth International Solar Wind Conference, AIP Conference Proceedings vol. 1216, 667

de Toma, G., Gibson, S. E., Emery, B. A., \& Arge, C. N., 2010b, SOHO 23: Understanding a Peculiar Solar Minimum, ASP Conference Series vol. 428, 217

de Toma, G. 2010, Solar Phys., doi 10.1007/s11207-010-9677-2

Harvey K. L. \& Recely F., 2002, Solar Phys., 211, 31

Emery, B. A., Richardon, I. G., Evans, D. S., \& Rich, F. J., 2009, J.A.S.T.P., 71, 1157

Emery, B. A., Richardson, I. G., Evans, D. S., Rich, F. J., \& Wilson, G. R., 2010, Solar Phys., doi 10.1007/s11207-011-9758-x,

Fisher, R. R., Lee, R. H., MacQueen, R. M., \& Poland, A. I, .1981, Applied Optics, 20, 1094

Gibson, S. E., Kozyra, J. U., de Toma, G., Emery, B. A., \& Onsager, T., Thompson B. J., 2009, J. Geophys. Res., 114, A09105

Gibson, S. E., de Toma, G., Emery, B. A., Riley, P., Zhao, L., Elsworth, Y., Leamon, R. J., Lei, J., McIntosh, S., Mewaldt, R. A., Thompson, B. J., \& Webb, D., 2011, Solar Phys., in press

Issautier, K., Le Chat, G., Meyer-Vernet, N., Moncuquet, M., Hoang, S., MacDowall, R. J., \& McComas, D. J., 2008, GRL 35, L19101

Jian, L. K., Russell, C. T., \& Luhmann, J. G., 2010, Solar Phys., doi 10.1007/s11207-011-9737-2

Judge, P. G., Burkepile, J., de Toma, G., \& Druckmüller, M., 2010, SOHO 23: Understanding a Peculiar Solar Minimum, ASP Conference Series vol. 428, 171

Lee, C. O., Luhmann, J. G., Zhao, X. P., Liu, Y., Riley, P., Arge, C. N., Russell, C. T., \& de Pater, I., 2009, Solar Phys., 256, 345

Lei, J., Thayer, J. P., Wang, W., \& McPherron, R. L., 2010, Solar Phys., doi 10.1007/s11207010-9563

Luhmann, J. G., Lee, C. O., Li, Y., Arge, C. N., Galvin, A. B., Simunac, K., Russell, C. T., Howard, R. A., \& Petrie, G., 2009, Solar Phys., 256, 285

McComas, D. J., Ebert, R. W., Elliott, H. A., Goldstein, B. E., Gosling, J. T., Schwadron, N. A., \& Skoug, R. M., 2008, GRL, 35, L18193

McIntosh, S. W., Leamon, R. J., Hock, R. A., Rast, M. P., \& Ulrich, R. K., 2011, ApJ Lett., 730, L3

Petrie G. J. D., Canou, A., \& Amari, T., 2010, Solar Phys., doi 10.1007/s11207-010-9687-0

Riley, P., Lionello, R., Linker, J. A., Mikić, Z., Luhmann, J. G., \& Wijaya, J., 2010, Solar Phys., doi 10.1007/s11207-010-9698-x

Schrijver, C. J., Livingston, W. C., Woods, T. N., \& Mewaldt, R. A., 2011, J. Geophys. Res., 38, CiteID L06701

Sheeley, N. R. 2008, ApJ, 680, 1553

Smith, E. J. \& Balogh, A., 2008, GRL, 35, L22103

Svalgaard, L. \& Cliver, E. W., 2007, ApJ Lett., 661, L203

Thayer J. P., Lei J., Forbes J. M., Sutton E. K., \& Nerem S. M., 2008 J. Geophys. Res., 113, CiteID A06307

Tokumaru, M., Kojima, M., Fujiki, K., \& Hayashi, K., 2009, GRL, 36, CiteID L09101

Tokumaru, M., Kojima, M., \& Fujiki, K., 2010, J. Geophys. Res., 115, CiteID A04102 
Tsurutani B. T., Echer, E., Guarnieri, F. L., \& Gonzalez, W. D., 2011, J.A.S.T.P., 73, 164

Wang Y. M., Robbrecht E., Sheeley N. R., 2009, ApJ, 707, 1372

\section{Discussion}

JON LINKER: Is it possible from X-ray and EUV emission to look at the area of coronal holes and to add up the coronal hole magnetic flux to determine the difference between the past minima?

Giuliana DE TOMA: Yes, we have not done it yet but this can be done combining coronal hole maps with synoptic magnetic maps. One important caveat is that to best observe the poles we need a favorable b-angle, so we cannot have a good view of both poles at the same time. Within the limits of how accurate coronal hole maps are, the flux balance for the open field can be determined and it is something worth doing.

LeIf SvalgaARD: Comment: The current minimum is very similar to the one of 100 years ago. This would suggest to me that TSI was also similar.

GiUliana DE TOMA: I agree.

ERIC PRIEST: You talked about the spectrum of solar wind speeds. Can the differences in velocity in the solar wind be explained by how coronal holes spread with height?

Giuliana DE Toma: Yes, it can. The very low speeds seen at the Earth in 2009 were because the solar wind during this time was coming mostly from the edges of the polar coronal holes that were smaller than in 1996, so there was a larger expansion factor.

Eugene Rozanov: Why do you compare 2008 and 1996? Perhaps 1996 is better comparable with 2009.

Giuliana De Toma: It depends on which observables you compare. At the photosphere, we do not see significant magnetic flux emergence in 2008 and 2009. Already in 2007, sunspot activity level was below the level observed in 1996. However, in the corona and heliosphere, there is a slow evolution throughout 2007-2009, with the solar wind reaching the lowest values in speed and magnetic field in 2009. This delay is not just in the Cycle 23 minimum but was also noticed during previous minima. The heliosphere tends to reach minimum conditions later than the photosphere.

Arnab Choudhuri: You are a co-author in one highly cited paper on solar cycle predictions. Theoretical issues were left out in your talk. What sort of lesson can the dynamo theorists learn from these observations?

Giuliana De Toma: This was the first time that physical models of the Sun were used to make solar cycle predictions. Because the predictions were so different, Cycle 24 will give us the opportunity to discriminate between low-diffusivity and high-diffusivity models and to test which class of models works best. This will help to constrain some important ingredients of the solar dynamo for which we do not have direct observations. It will be interesting to see what happens with Cycle 24. Of course, there is the possibility that Cycle 24 will end up being an average cycle, in which case it will be difficult to say anything conclusive about the two classes of models. Right now, we see a strong asymmetry between the two solar hemispheres. If this continues, it is possible that we 
will have a strong cycle in the northern hemisphere and a weak cycle in the southern hemisphere. This asymmetry will also be interesting to explain for dynamo modelers.

DAVID WEBB: This asymmetry is very interesting. How does this compare to previous cycles?

Giuliana DE Toma: There seems to be a time lag between the two hemispheres. This has been going on since 2006, longer than we have have observed in previous cycles.

SACHA BRun: Comment: There is evidence that during the Maunder minimum the southern hemisphere was more active. We looked at this in detail. We find that when the dipole and quadrupole have the same size, one hemisphere is more active. So clearly the dipole vs. quadrupole amplitude plays a key role in this asymmetry.

SAcha Brun: Since sunspot number was so much smaller in 2008 than in 1996, how do explain the complex magnetic topology? What features modify the global field?

Giuliana DE TOMA: What is important for the coronal structure is ultimately the balance of the magnetic flux at high and low latitudes. During the recent minimum, the low-latitude magnetic flux was weak, but so were the polar magnetic fields. In 2007 and 2008, the relative strength of the polar dipole relative to the equatorial dipole, the quadrupole and the higher order moments was lower than in 1996 or 1986 and this is what allowed the corona to retain some complexity. In 2009, as magnetic flux at low latitudes decayed, the polar dipole moment became more prominent but never as important as in 1996.

SACHA BRUN: You said that sunspot number is not a good indicator for the solar corona. Is there another index that we can use?

Giuliana DE Toma: This is a hard question. I do not know if there is a good proxy for the corona. To infer the coronal structure, you really need the 3D magnetic field distribution since the organization of the field spatially is as important as the strength of the field for the corona and heliosphere.

JÜRGEN SChMitT: You pointed out that the last sunspot minimum was not unusual compared to previous minima around 1900 and should not be confused with a Maunder minimum. So how would the Sun look like when it is really in a Maunder Minimum and how does this differ from what we saw in 2008-2009?

Giuliana DE Toma: Nobody can know for sure what the Sun looked like during the Maunder minimum. However, there is increasing evidence from the comparison with other stars in grand minima that solar irradiance was not very different during the Maunder minimum from what we observe during a normal minimum. The work of Schrijver and co-authors also points into this direction. In 2008 and 2009, the magnetic flux on the Sun had enough time to diffuse to a smaller scale to give us some idea of how the Sun would look like after a long period of inactivity. As argued by Schrijver et al., the fact that TSI was about the same in 2008-2009 as it was during the previous minima, suggests that the Sun was not much dimmer during the Maunder minimum. 